\title{
Implementation of an IEEE 802.6 Compliant Card for the ISA Bus
}

Lakshman Narayanswamy, Jasvinder Singh, Keerthi Mitra Umesh Kulkarni, Anurag Kumar, Utpal Mukherji ERNET Project

Department of Electrical Communication Engineering Indian Institute of Science e-mail:\{laks,jassi,umesh,anurag,utpal\}@ece.iisc.ernet.in

\begin{abstract}
This paper describes an implementation of an IEEE 802.6 compliant, DQDB card for the PC-AT bus. We discuss the various issues involved in design of the card and describe the various functional blocks of the card in detail. We also discuss how a DQDB network can be used as a distributed multiplexer for an ATM link.
\end{abstract}

\section{Keywords}

IEEE802.6, DQDB, MAN, ISA, ATM multiplexer, high-speed network

\section{INTRODUCTION}

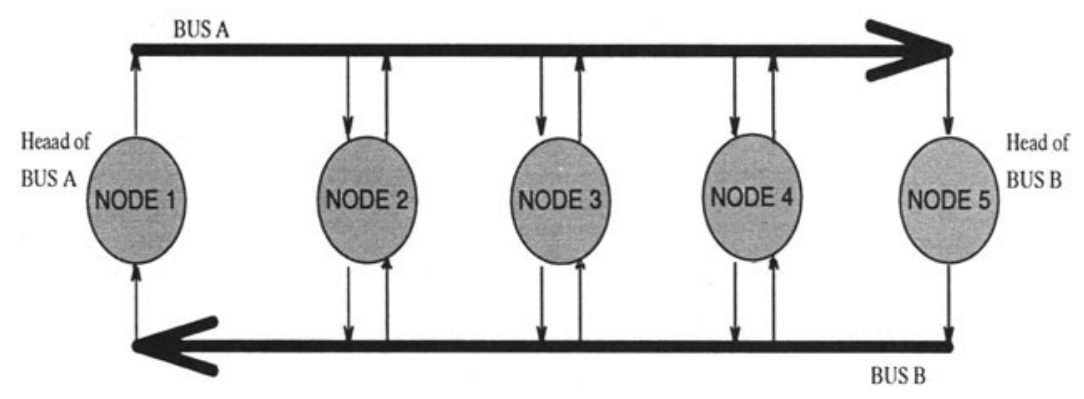

Figure 1: DQDB Network Topology.

Rapid growth of local area networks (LAN) which span a limited distance have given rise to the need for interconnecting LANs. Metropolitan area networks (MAN) that span distances greater than that of LAN and can meet 
the bandwidth requirements for the interconnection of many LANs are ideally suited to meet this need. The IEEE 802.6 MAN standard for a Distributed Queue Dual Bus (DQDB) network defines a high speed common channel access protocol over dual unidirectional buses. The topology of the DQDB network is as shown in Fig. 1. We briefly describe the media access protocol. With reference to Bus A and any of the intermediate nodes in Fig. 1, nodes to the left are called upstream nodes and nodes to the right are called downstream nodes. With respect to Bus B, it is vice-versa. The structure of the DQDB cell is as shown in Fig. 2. The head of bus node for both buses generates cells at regular intervals that are sized to transport fifty-three octets. The MAC protocol uses the Busy bit and the Request bit of the Access Control Field to gain access to the bus. We describe the procedure for a node to transmit data on Bus A. When a node has cells to transmit on bus $\mathrm{A}$, it sets the request bit of a cell passing on Bus $B$. This set request bit is noted by all downstream nodes by which the cell passes and a counter which indicates the number of requests that have passed by on bus B is incremented. This is the request counter; at a given node the count value held by this counter indicates the number of outstanding requests made by upstream nodes. To transmit a cell, the node copies the value of the request counter into a countdown counter. The value held by the countdown counter indicates the number of outstanding requests that have to be serviced before the node can transmit its cell. For every cell that passes by on Bus A without the busy bit set, the value of the countdown counter is decremented. When the countdown counter value reaches zero, the node transmits in the next available free cell. A node at any given time can have only one outstanding request per bus. Detailed description of the DQDB protocol are presented in [Newman et al.,1988], [Newman et al.,1986], [DQBD Draft]. Reference [Pillai et al.] reviews the performance of the DQDB MAC protocol.

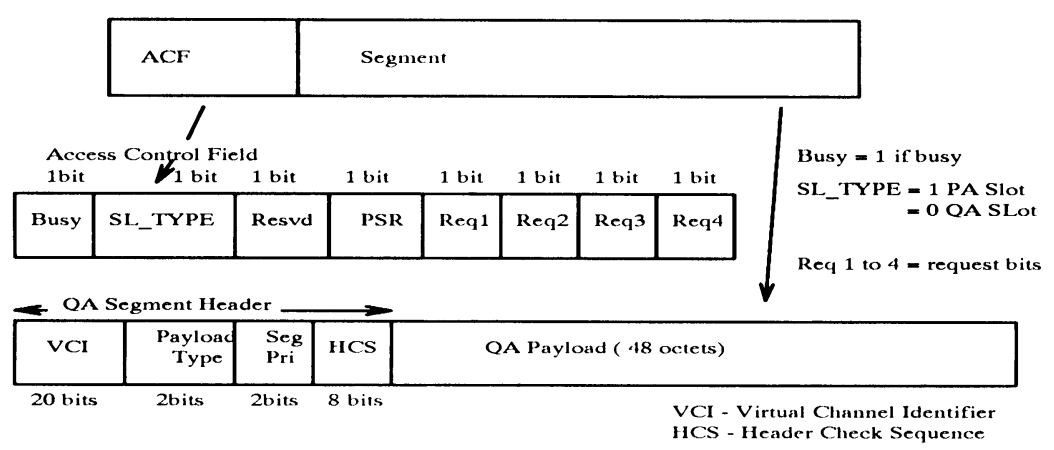

Figure 2: DQDB cell structure.

DQDB provides for a range of services that include connectionless data transfer, connection oriented data transfer and isochronous services. DQDB provides these services by using the Queued Arbitrated (QA) and Pre-Arbitrated (PA) access methods. The QA access provides for the asynchronous transfer of cells using the distributed queue access protocol. The QA function supports four 
priority queues for the connectionless and connection-oriented data services. The PA function provides for connection oriented transfer over a guaranteed bandwidth. The PA functionality requires the establishment of a connection, for which a Virtual Connection Identifier (VCI) value is assigned.

We have undertaken to prototype a DQDB network for attractive reasons. This exercise is part of an overall effort at building a broadband networking testbed. The DQDB protocol provides a degree of fairness in medium access by use of a distributed queue, and offers a mechanism for supporting isochronous services. The second attractive feature of DQDB is the close alignment of its cell structure with that of the Asynchronous Transfer Mode (ATM) cell structure. The goal of accommodating diverse source characteristics in B-ISDN is to be met using ATM. DQDB can be used to provide the function of multipoint access to an ATM link.

\section{$1.1 \quad$ Related Work}

Reference [IEEE JASC] is devoted to high-speed computer-network interfaces. Reference [Davie] and [Traw et al.] discuss the design of an ATM network interface for high speed workstations, the lessons are relevant to the design of the DQDB network interface, as the cell structure of DQDB and ATM are similar. Paper [Davie] describes a network interface for a $622 \mathrm{Mb} / \mathrm{s}$ ATM link using microprocessors to implement transmit and receive functions. This card is designed for a DEC workstation whose I/O bus supports a peak bandwidth of 800 $\mathrm{Mb} / \mathrm{s}$. Reference [Traw et al.] describes a network interface for connecting to a SONET based ATM link at the rate of $155 \mathrm{Mb} / \mathrm{s}$. This interface is designed for a RISC 6000 workstation which supports a peak I/O bus bandwidth rate of 320 $\mathrm{Mb} / \mathrm{s}$. This implementation is done using dedicated hardware. Segmentation and reassembly is done by the network interface card so that the number of buffer copies required is reduced and the high I/O bus bandwidth is effectively utilized.

This paper is organized as follows. Section 2 discusses the issues that influenced the design of the DQDB add-on card. Section 3 describes the various blocks that make up the DQDB card implementation and Section 4 charts the future direction that is planned for the project. Section 5 concludes the paper.

\section{DESIGN ISSUES}

Within the framework of the OSI reference model, the IEEE 802.6 standard [DQBD Draft] defines the distributed queue protocol and a convergence function (also referred to as DQDB layer) that provides connectionless MAC service access point to the Logical Link Control sublayer. The VCI value provides a means to identify the service to which the cell belongs. The VCI value of all bits set to one is the default value for the connectionless MAC service provided by the convergence function. The DQDB layer receives the Mac Service Data Unit (MSDU) for transmission from the LLC sublayer and forms an Intermediate Mac Protocol Data Unit (IMPDU) by the addition of a header and trailer. The important fields of the header are the source address, destination address, 
length and a unique tag. The trailer is made up of the same length and the unique tag. The IMPDU is segmented into fifty-two octet cells which is made up of four octets of QA header, four octets of DMPDU header and trailer and forty-four octets of payload. Fig. 3 diagrams the segmentation process which is described in section 3. On the receiving node the DQDB layer receives fiftytwo octets from the bus, checks to see if the destination address matches and reassembles the forty-four octet payloads to form an IMPDU which is handed to the LLC sublayer.

The service offered by a network interface adapter to the host is dictated by the host architecture. For maximum flexibility of the network adapter card, the obvious design choice is to use a microprocessor to implement the card functionalities. After having carefully studied this option, we find that none of the available CISC processor or microcontrollers satisfied the timing requirements. In a typical microprocessor based design, the processor is informed of external events (e.g., arrival of a new cell) by means of a hardware interrupt. In such a design, the interrupt latency time of the microprocessor should be less than the interarrival time of the external event (in a $43.776 \mathrm{Mbps}$ DQDB network, the cells interarrival time is $10.42 \mu \mathrm{s}$ ) in order that all events be processed. We find locally available microprocessors and microcontrollers are not suitable as their interrupt latency time is in the order of milliseconds. Hence we have implemented all time critical functionalities in hardware.

The issue of whether the DQDB layer should be implemented on the card or on the host can be resolved by considering the capabilities of the host. The DQDB card that we describe is designed for the PC-AT bus. The PC-AT platform was chosen for its ease of availability at affordable cost. The main constraints while designing for the PC-AT bus are the maximum data rate of the bus, mapping of the card memory to the host memory, and the physical dimension of the card that can be accommodated by the PC-AT platform. We take the worst case condition of having to receive all the cells on both the buses and calculate the time available to process each cell. The DQDB network that we have designed operates at $43.776 \mathrm{Mbps}$. Every $125 \mu$ s the head of bus node generates twelve, fifty-seven octet slots. The first four octets are management octets, the fifth octet is the Access Control Field used by the DQDB MAC. The rest of the fifty-two octets form the segment payload. At $43.776 \mathrm{Mbps}$ the per slot timing is $10.41667 \mu \mathrm{s}$. Considering both the buses, the time available to process a slot is $5.21 \mu \mathrm{s}$. For the DQDB layer to be implemented on the host, the host should be able to copy the incoming cells into host memory at the maximum input rate. This requires a host bus transfer rate of $(52 / 5.26 \mu \mathrm{s})=9.886 \mathrm{MBps}$. The maximum bus transfer rate using the byte DMA mode of the PC-AT bus is $1 \mathrm{MByte} / \mathrm{sec}$ [Solari]. ${ }^{1}$ Hence, the host is not capable of copying the cells at the maximum input rate from the buses. The other alternative is to provide buffering on the card. The incoming cells destined to the node are copied into

\footnotetext{
${ }^{1}$ The exact DMA throughput is a function of: the length of the instruction cycle that is interspersed with the DMA cycle, higher priority DMA request that are queued to be serviced and the DRAM refresh cycle every $15 \mu \mathrm{s}$. We have assumed the DMA rate to be $1 \mathrm{MB} / \mathrm{s}$ given that the maximum input clock to the DMA controller can be $5 \mathrm{MHz}$ and that we use the single byte DMA transfer mode.
} 
the buffer and the host interrupted. The host then copies the fifty-two octet cells from the buffer on the card for further processing. The disadvantage of interrupting the host for every fifty-two octet cell is the high overhead associated with the processing of a hardware interrupt.

Our design overcomes the low bus data transfer rate and high overhead associated with interrupt processing by having a buffer on the card and reassembling the received cells into IMPDU's in the buffer. The host is not interrupted for every cell, but only after the complete IMPDU has been reassembled. Our design supports a maximum IMPDU size of 6 KBytes. An important consideration in implementing the receive function of the DQDB layer is the number of IMPDU's that can be concurrently assembled. Our design provides for concurrent reassembly of five MID's and maximum IMPDU size of 6 KBytes. We feel that support for five concurrent reassemblies are sufficient for a PC-AT host running a multi-tasking operating system (e.g. UNIX) to support popular networking services (e.g. file transfer, remote login ..). Support of every additional reassembly requires additional logic and hence board space, which is a scarce resource in our implementation.

The DQDB layer transmit function accepts MSDU's from the LLC sublayer, segments them into forty-four octet payload and adds the header to form a fiftytwo octet cell which is then queued for transmission. To resolve the issue of whether to implement the transmit function of the DQDB layer on the card or on the host, we examine the times involved in transmission of a IMPDU. They are, the processing time required to calculate the header and the trailer to form a fifty-two octet cell, the time required to copy data from the host memory to the card and the time the cell remains queued before it is transmitted onto the network. The third component is an attribute of the network load and not a function of the host-card architecture. The other two times are the processing delay and the time taken to copy data from the host memory to the card. The minimum time required to copy data from the host memory to the card is calculable from the maximum data rate of the PC-AT bus. Assuming a maximum I/O channel rate of $1 \mathrm{MB} / \mathrm{sec}, 52$ octets can be transferred from the host memory to the card in $49.5 \mu \mathrm{s}$. A 80386 processor with a clock of $25 \mathrm{MHz}$, can execute 309 instructions in $49.5 \mu \mathrm{s}$ if the average instruction cycle is taken to be 4 machine cycles. We argue that an optimized implementation of the segmentation process will take lesser time than that required for copy of the cell from the host memory to the card. Following the above, we can say that there is little gain in implementing the processing required for segmentation of a IMPDU on the card in hardware, since the bottleneck in using the complete bandwidth of the DQDB bus is not the CPU processing speed of the host, but its limited I/O bus speed. Our design assumes that the segmentation is done by software on the host. A nineteen cell FIFO buffer is provided on the card into which the host writes the cells to be transmitted. A significant part of the trailer calculation for every cell is the CRC. To cut down on the processing time required to calculate the $\mathrm{CRC}$, we have implemented CRC calculation in hardware.

Calculation of the 8-bit and 10-bit CRC requires the 3 octet QA header and the 46.75 octet ( 46 octets and 6 bits) payload to be serialized and fed to the 8 - 
bit and 10-bit CRC generator. The transmit CRC calculates the remainder and appends 8 bits to the QA header and 10 bits to the QA payload. The receiver calculates the CRCs of the incoming cell and checks for zero remainder. CRC calculation on the transmit and the receive path are ideally suited to be done parallely as the octets are read into the transmit and receive FIFO from the host and network respectively. On the receiver, as the incoming octets are read from the network and copied into the receive FIFO, the octets are also copied to a shift register. The clock to the shift register should be at least eight times as fast as the incoming octet rate of $5.472 \mathrm{MBytes} / \mathrm{s}$ so that all eight bits of the octet are shifted to the CRC calculator before the next octet arrives from the network. On the transmitter, since the host transfers 16 bits over the I/O bus, the clock to the shift register should be at least 16 times as fast as the data transfer rate from the host to the transmit FIFO.

To reassemble five IMPDUs of 6 KBytes each, a 30 KByte buffer is required. Further, buffer for copying SSM segments has to be provided. To satisfy the above requirement we have chosen to use $32 \mathrm{Kx} 8 \mathrm{bit}$ dualport RAM as the buffer which can accomodate five IMPDUs and forty-two SSM segments. The choice of dualport RAM is obvious since there are two functional blocks that need access to the RAM. One is the Receive block that writes the incoming cells into the RAM. The other is the host, which reads the assembled packet from the DQDB card buffer into the host memory. One option would have been to use a single port RAM and give priority to the receive block to write the incoming cells into the RAM. This strategy requires the host to wait till the receive block gives up access to the RAM and then transfer the packet from the RAM to the host memory. This method is not acceptable as it increases the host-to-card data transfer time, which is the bottleneck in our design. Use of a dualport RAM solves this problem by providing simultaneous and independent access to the RAM for both the host and the receive block.

A node on the DQDB network has access to the optical fiber bus by means of an active or passive read and write connection. Writing on to the bus then is a logical OR of data from the node and data from upstream. We have implemented the bus as segmented cables between successive nodes. The option of implementing a bus using optical couplers is not flexible for addition of nodes to the network. Each DQDB interface card has two optical receivers and two optical transmitters, one transmit and receive pair for each bus. For each bus, each node on the DQDB network has an optical fiber coming in to a receiver and and an outgoing fiber connected to the corresponding transmiter.

To optimise on the board space and to meet our timing and power requirements, we have implemented most of our hardware logic using ALTERA's Programmable Logic Devices [Altera Databook].

\section{DESIGN DESCRIPTION}

We have implemented the connectionless service using the QA convergence function provided by the DQDB MAC to the logical link sublayer. The functionality of the DQDB layer for the connectionless protocol is described in detail with reference to Fig. 3. 
Fig. 3 shows the construction of the IMPDU from the MSDU by the addition of header and trailer. The IMPDU is then segmented into fifty-two octet DMPDUs and queued for transmission. The QA segment transports the 48 octet DMPDU as its segment payload. The receive block does the reassembly of DMPDUs to get back the IMPDU. The reassembly is done by associating one MID (message identifier) value to the entire IMPDU. This MID value is contained in the DMPDU header of every cell that contains the IMPDU's data as its payload. Hence for the interval of time that all the DMPDUs from an IMPDU are received, the MID value associated with that IMPDU is unique across the DQDB network. The first DMPDU derived from the IMPDU is the Begin-
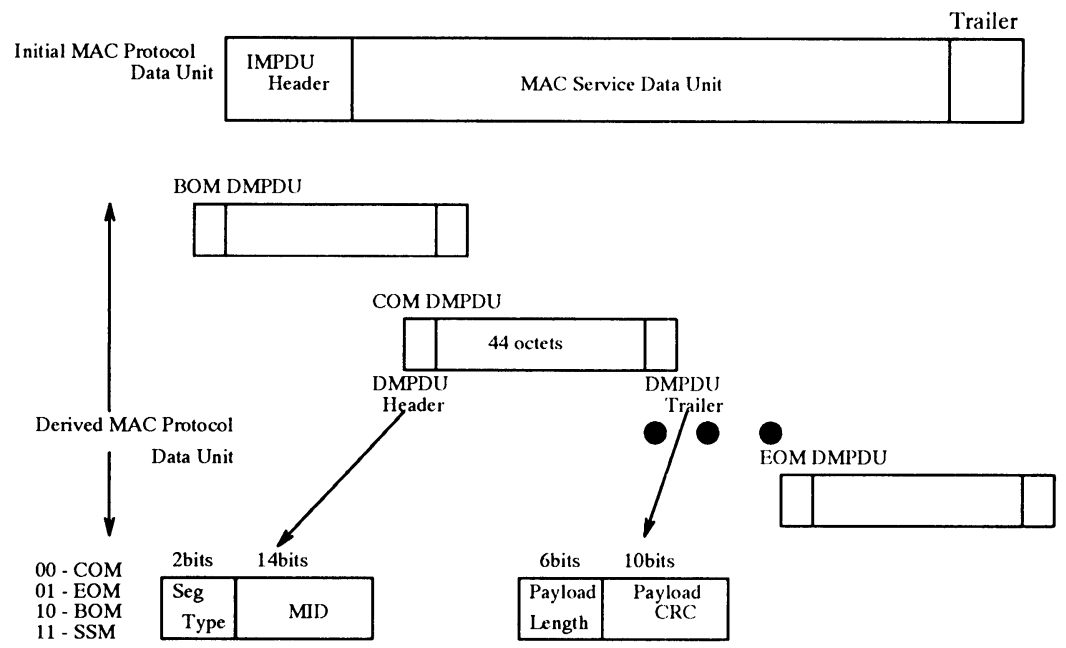

Figure 3: DMPDUs from IMPDU.

ning of Message (BOM). The BOM has both the destination node address and the MID value associated with the IMPDU being transferred. On the arrival of a BOM, the receive block checks for a destination address match, if there is a match, the MID value contained in the BOM cell is stored. Subsequent DMPDU constructed from the IMPDU being transferred are Continuation of Message (COM) which contain the MID value as part of the DMPDU header. If the received COM cell's MID value matches, then the cell is copied into the RAM. The last DMPDU derived out of the IMPDU is the End of Message (EOM). If the received EOM cell's MID matches with one of those being assembled, the cell payload is copied into RAM and the MID value removed from the list of MIDs being reassembled. Further, no cell containing the MID value will be copied until the receiver gets a fresh BOM with the same MID value. To maintain uniqueness of the MID value, the DQDB standard defines allocation of MID values through the management octets in the overhead part of the DS3 frame. Our design statically allocates a set of usable MID values to each node. 


\subsection{Physical Layer Block Design}

This section describes the physical protocol block implementation. The block diagram of this layer for one of the buses is as shown in Fig. 4.

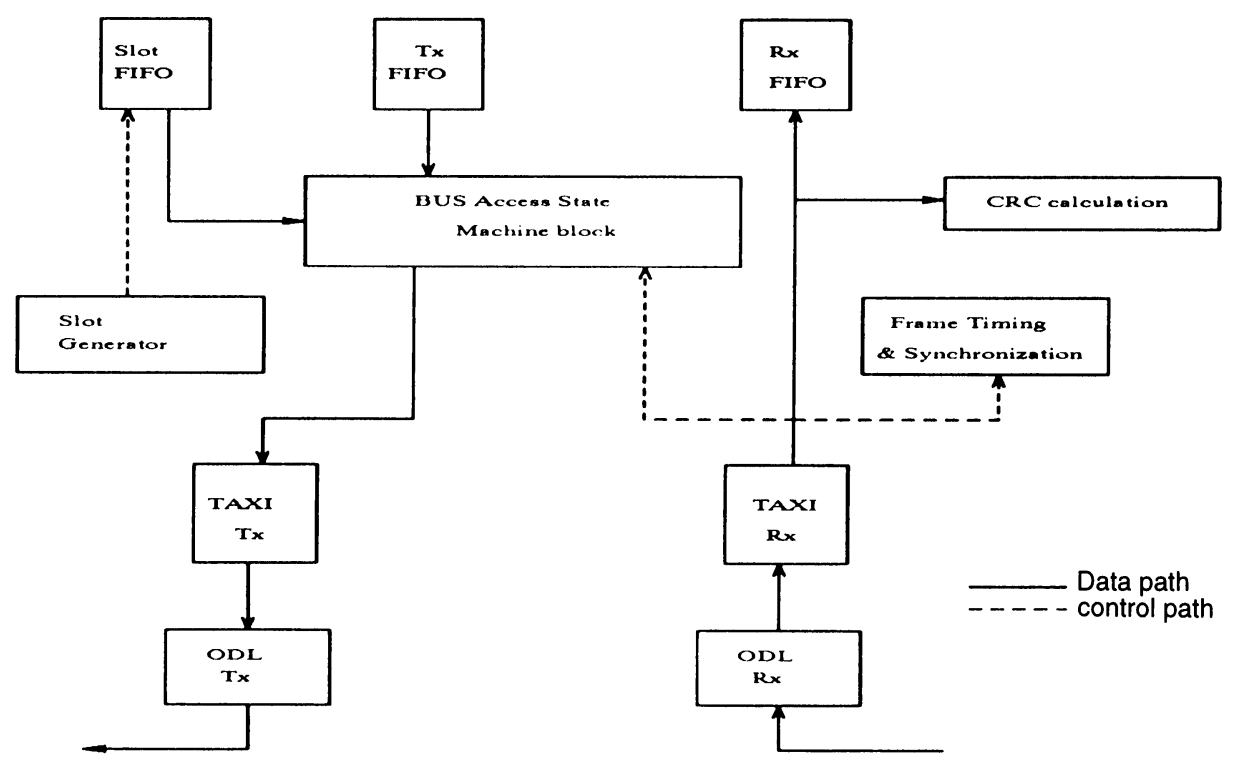

Figure 4: Physical Layer Protocol Block

The DQDB standard recommends mapping of the DQDB physical layer to the DS3 carrier. The DS3 signal consists of 699 octets per $125 \mu \mathrm{sec}$ time period. One bit in 85 bits is used for the DS3 overhead, which leaves 690.78 octets for the information payload. We have closely alligned our physical layer to the DS3 signal, though we do not implement the DS3 overhead bits. We use a $125 \mu \mathrm{s}$ frame containing 12 slots at the rate of $43.776 \mathrm{Mb} / \mathrm{s}$. Each slot contains 57 octets. The information payload is $57^{*} 12=684$ octets in $125 \mu \mathrm{sec}$, hence each slot time is $10.41667 \mu \mathrm{sec}$. The first two octets, A1 and A2 are slot delineating octets which are assigned hexadecimal values of F6 and 28. The next two octets are the path overhead octets. The first four octets are used by the physical layer management entity. We use the term cell to denote the 52 octets that follow the four management octets in a slot and the Access Control Field (ACF) whose fields are as shown in Fig. 2. We have chosen to implement only one of the four queue priorities provided by the four bit request field.

The data path in the physical layer block is traced in Fig. 4. Reception and transmission of signals to/from the optical fiber is done using AMD's Transparent Asynchronous Xmitter Receiver Interface (TAXI) [TAXI Datasheet] and AT\&T's Optical Data Link (ODL) [ODL Datasheet] chips. For transmission, the transmit TAXI serializes the 8-bit parallel data at the input and outputs 10-bit ECL signal units to the Tx-ODL. For reception, the $\mathrm{Rx}-\mathrm{ODL}$ converts the optical signal into ECL level electrical signal which is fed to the $\mathrm{Rx}$-TAXI serial input. The output of the Rx-TAXI is the 8-bit parallel data units at TTL signal level. 
The transmit TAXI does a $4 / 5$ encoding of the data presented to its parallel input. The serial output rate of the TAXI is $n$ times the input rate of the clock to the TAXI. The factor $n$ depends on the width of the parallel input data to the TAXI. For 8 bits wide input data the factor $n$ is 10 . To achieve the line rate of $43.776 \mathrm{Mbps}$ the input clock to the TAXI should be $43.776 *(5 / 4) *$ $(1 / 10)=5.472 \mathrm{MHz}$. Hence, on every rising edge of the clock of $5.472 \mathrm{MHz}$, a byte of data has to be given to the Tx TAXI. Correspondingly the receiver TAXI decodes the $4 / 5$ encoded serial input and gives a 8 bit parallel output every $182.75 \mathrm{nsec}$.

A card can be configured as Head of Bus (HOB) or an intermediate node. For the bus for which it is the head node, the data path is from the slot FIFO to the Tx TAXI. The HOB node generates and transmits on the bus, twelve, fifty-seven octet slots every $125 \mu$ sec. As part of the initialization of the HOB node, the host software writes 12 slots of fifty-seven octets into the slot FIFO. Each of the slots starts with two framing octets and two management octets and fifty-three zero valued octets representing the fifty-three octet cell. The slot generator in the HOB node reads sucessive slots from the slot FIFO and routes the octets to the Tx TAXI for transmission. This is repeated every 125 $\mu$ s providing a constant stream of slots on the DQDB bus. When the HOB node has data to transmit on the bus, the first five octets are read from the slot FIFO, the fifth octet is routed through the Bus Access State Machine block and the rest of the fifty-two octet cell is read from the Tx FIFO and written to the Tx TAXI.

Synchronization of the receiver to the start of each slot is done by the frame syncronization block. After the initialization of the card, consecutive octets are compared for the value of the A1 and A2 octets. If consecutive octets match with $\mathrm{A} 1$ and $\mathrm{A} 2$, the receiver is assumed to be synchoronized with the incoming slot. To maintain synchronization, the $\mathrm{A} 1$ and $\mathrm{A} 2$ patterns are matched at the start of every 57 octet slot.

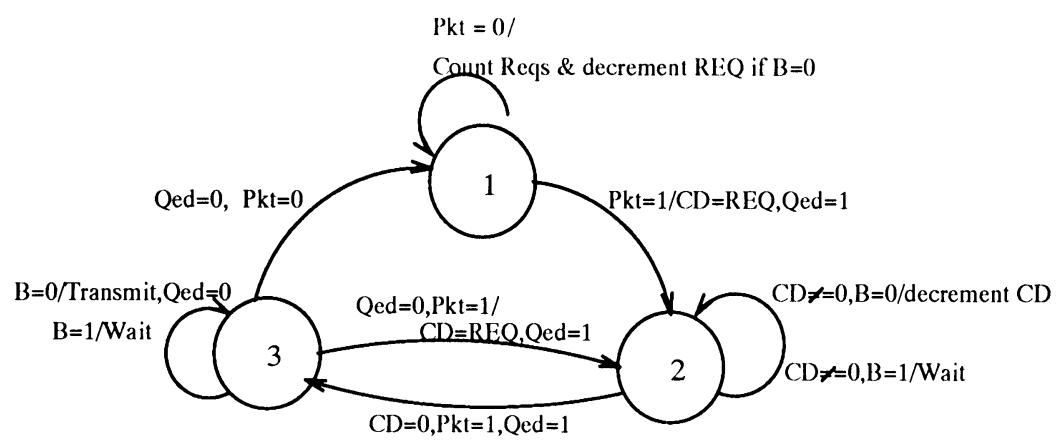

Figure 5: DQDB Bus Access State Machine

The DQDB MAC protocol is implemented as a state machine shown in Fig. 5 We describe the functioning of the state machine when a node want to transmit on bus A. When the node does not have anything to send, it is in state 1, the 
IDLE state. In this state the requests going on bus B are counted and for every empty slot that passes on Bus A, the request counter is decremented.

When the node has a cell to transmit, the variable Pkt is set to one and transition to the next state take place. During this transition, the contents of the Request counter are transferred to the Count Down counter and the request counter is reset to zero. Simultaneously, the variable called Q'ed will be set to indicate that the current cell is queued to be sent and a request will be sent on bus $\mathrm{B}$. If the current slot on bus $\mathrm{B}$ has the request bit already set, then this request will be held back and sent when the opportunity arises. Inability to send a request to transmit a cell does not prevent that cell from being queued and transmitted. In this condition the request counter will continue to count the requests on bus B but is not decremented. For every empty slot on bus A, the countdown counter is decremented. When the value of the counter reaches zero, transition to state 3 takes place.

State 3 in the state diagram is the transmit state. While in this state, if an empty slot arrives then the busy bit in the ACF is set to 1 and the cell is transmitted. If the busy bit is already set to 1 , the state machine waits till the next empty slot arrives. Once the transmission is complete, the Que'd and Pkt variables are reset to zero. If there is another cell to be transmitted, then the state machine moves to state 2 , otherwise it moves to state 1 , i.e. Idle state. All state changes are triggered by the arrival on the bus of the fifth octet in a slot, i.e. the ACF.

\subsection{Transmit Block Design}

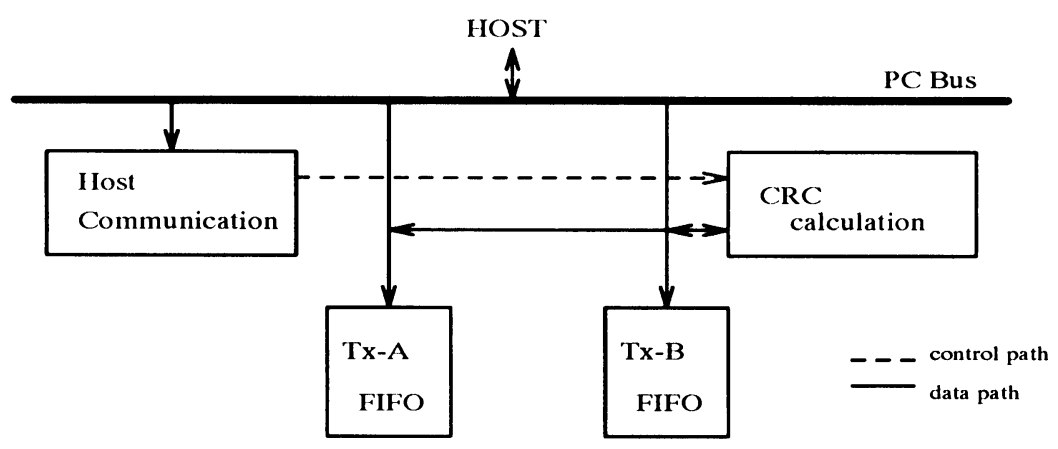

Figure 6: Transmit Block Diagram

This section describes the design of the Transmit machine of the DQDB network. Fig. 6 is the block diagram of the transmit block. As mentioned in Section 2, the DQDB layer that does the segmentation of the IMPDU is implemented in software on the host. After the DMPDU is created from the IMPDU payload, a routing decision is made as to which bus the cell is to be transmitted on. The host checks the transmit counter which holds the value of the number of cells queued to transmit for a particular bus. The $1 \mathrm{Kx} 8$ bit transmit FIFO (Tx-FIFO) can queue nineteen cells to be transmitted. If 
the transmit counter value equals nineteen, then the host has to wait for the transmit counter value to decrement, otherwise the host copies the DMPDU into the Tx-FIFO. The last ten bits of the DMPDU are the payload CRC. The host writes a 16 bit word to the I/O port on which the Tx-FIFO is mapped. The 16 bit word is split into two octets and written into the FIFO. In parallel the octets are fed to an $8 \mathrm{bit}$ shift/storage register that shifts the octets out serially and feeds the bits to a CRC block for the CRC calculation. At the end of the payload, the 10 bit CRC is appended along with a 6 bit payload length into the Tx-FIFO. The transmit counter value is incremented by one to indicate the number of cells queued for transmission. We have provided the option of copying the cell octets from the host memory into the transmit FIFO using the byte mode DMA or through a programmed loop involving the CPU.

\subsection{Receive Block Design}

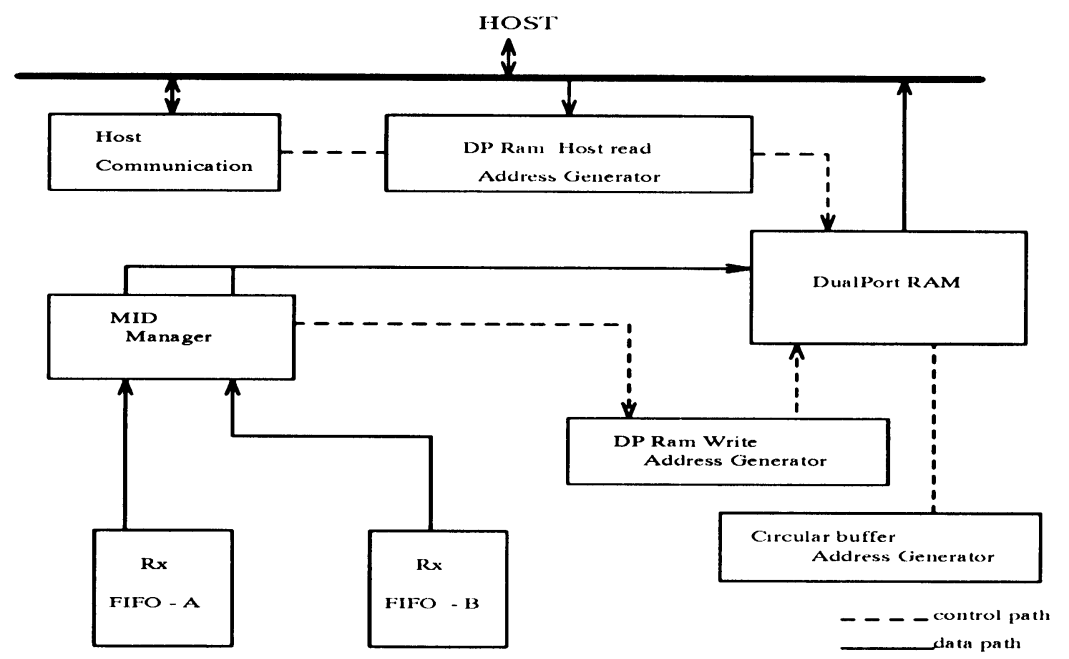

Figure 7: Receive Block Diagram.

All cells arriving on the bus with the Busy bit set to 1 are copied into the receive FIFO (Rx-FIFO) of the bus it has arrived on. The receive block's per cell processing time of $5.21 \mu \mathrm{s}$ (half the cell time) is required to handle the maximum cell arrival rate on both the buses. After copying a complete cell from the bus into the Rx-FIFO, the physical layer block increments a counter which indicates the number of cells queued in it. The receive block reads octets out of the FIFO and processes them. The MID management block manages the MIDs that are being reassembled. Our design supports concurrent reassembly of five MIDs. The MID values being reassembled are stored in latches which are compared for an MID match with the sixth octet of a cell being read out of a Rx-FIFO. When the DMPDU segment type is BOM and if one of the MID latches is free, the MID value associated with the incoming BOM cell is copied into the available MID latch. The destination address, which is the eighteenth 
octet of the incoming cell, is compared for a match with the address of the node. If the address does not match, the reassembly is stopped and the MID latch into which the incoming MID value was copied is reset. Memory management of the Dual Port RAM is done by statically assigning six KBytes of dualport RAM to each MID value that is being reassembled. Hence the LLC sublayer can submit to the DQDB layer a six KByte MSDU for transmission.

The address generation block generates the address into which the received octets corresponding to a particular MID are written into the RAM. When an incoming MID value matches, it enables the address generation counter associated with that particular MID latch. Hence cells with the same MID value are copied contiguously in the RAM. After the EOM cell for a particular MID is copied into the RAM, the MID value is reset. The host is communicated by means of an interrupt when the EOM cell associated with a particular MID is copied and the complete IMPDU is now available in the RAM. On being interrupted the host reads the status register to know where in the RAM the reassembled IMPDU can be copied from.

Single Segment Message (SSM) are cells that fit a whole IMPDU within a DMPDU. On receipt of an SSM, the receiver checks the destination address (DA) of the received cell for a match. If the DA address matches and there is memory available in the RAM, the cell is copied into the dualport RAM. The transfer of SSMs from the network to the host is by using 2 KBytes of the dualport RAM as a circular buffer. The write pointer to the circular buffer is incremented when an SSM for which the DA address matches is copied into the RAM. The read address of the circular buffer moves as the hosts reads off SSMs from the dualport RAM. The ssm-count counter keeps count of the number of SSMs present in the circular buffer. This counter is readable by the host through a I/O port. The host is interrupted only when the maximum number of SSM cells that can be accomodated in the circular buffer is reached. The host is not interrupted for every received SSM, but is expected to find out the number of SSM cells in the buffer by polling the ssm-count register.

An incoming cell, whose MID value matches with one being reassembled but whose CRC does not match is discarded and the corresponding reassembly is aborted. Another event which aborts reassembly of an MID and causes an MID latch to be set free is the expiry of the MID timer. There is a timer associated with each MID being reassembled. This timer sets an upper limit on the time that can elapse between the receipt of consecutive cells with the same MID value. The timer expiry value is set to 0.7 seconds as recommended by the DQDB standard.

The host communication block facilitates communication between the host and the card. The dualport RAM memory on the card is mapped on to an $\mathrm{I} / \mathrm{O}$ ports of the host. There is a read address counter on the card that the host loads by writing to a I/O port. Read operations on the I/O port serve as a clock to the read address generator, and increment the address the RAM is read from. The read address also enables the dualport RAM; hence data in the DP Ram corresponding to the address from the read address generator is available on the $\mathrm{PC}$ bus. 


\section{FUTURE WORK - DQDB AS AN ATM MULTIPLEXER}

To be able to provide distributed access to a high speed ATM link a multipoint to single point access function is required. DQDB fits in very well to serve this need due to the close alignment of the DQDB and ATM cell structure. Reference [Takase at al.] describes an experimental B-ISDN system in which DQDB is used to multiplex cells to an ATM link. Reference [Byrne et al.] provides a good overview of the evolution of MAN to B-ISDN. Reference [IEEE Network] is a collection of articles on the deployment of ATM. Our effort at development

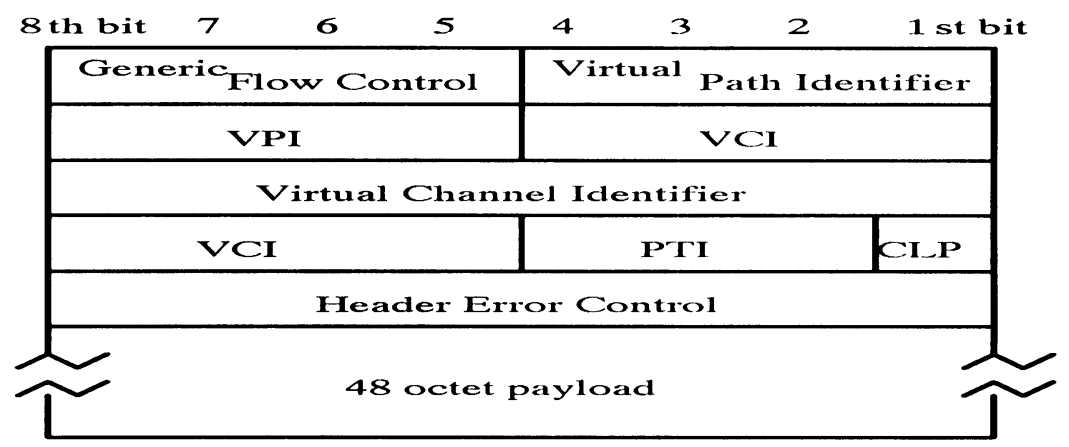

Figure 8: ATM cell structure.

of the DQDB card is proposed to be integrated into a B-ISDN test-bed, as a distributed access multiplexer. Our future work will be aimed at using DQDB to provide multipoint access to an ATM link. The ATM cell structure at the User Network Interface (UNI) is as shown in Fig. 8. A DQDB network can be connected to the $T_{B}$ interface of the B-ISDN connection architecture as shown in Fig. 9. The other method would be to connect a DQDB network to an ATM switch through a terminal adapter (TA).

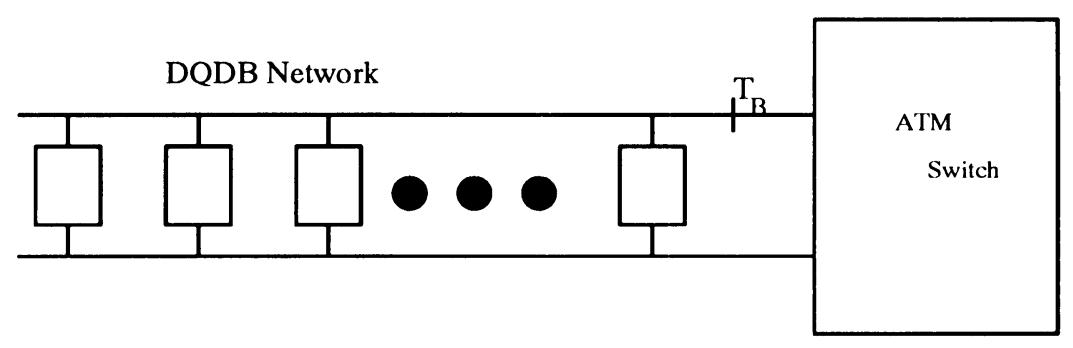

Figure 9: DQDB as an ATM link multiplexer.

To implement the first option, the DQDB and ATM headers have to match. The GFC field in the ATM header at the UNI is for providing multipoint access to a single physical link. While ATM provides for 4 bits at the UNI for the 
single to multipoint access protocol, $\mathrm{DQDB}$ requires the $8 \mathrm{ACF}$ bits for the implementation of the DQDB MAC protocol. If the VPI field at the UNI is not used and a 16 bit VCI is used instead of a 20 bit VCI as defined in the DQDB header, then the direct connection of the DQDB network to a ATM switch is possible. For common VCI values to be used across the DQDB network and the ATM switch, a common signalling protocol for VCI value allocation will have to be used amongst the DQDB nodes and the ATM switch. The connection methodology shown in Fig. 9 is suitable only for data traffic. To transport isochronous traffic between a DQDB network and an ATM switch, the DQDB network will have to be connected to an ATM switch through a terminal adapter (TA). We are studying the implications and feasibility of the above two options to use DQDB as a distributed multiplexer to a ATM link.

\section{CONCLUSIONS}

We have described the implementation of a DQDB network interface card. Though our card is designed for the PC-AT bus, it can be easily adapted to an EISA platform that provides a higher I/O bus transfer rate. Physical space limitation of the card has restricted the functionality that the card provides. We feel that it would be better to have two physical cards with the physical layer functionality on one card and the transmit and receive block functions on the second card. The card has been debugged and its functionalities shown working. Presently device driver and transport layer software to integrate the card to the host is being developed.

\section{ACKNOWLEDGEMENTS}

We would like to thank Dr. Bharat Doshi, AT\&T Bell Labs, Dr. T. R. Vishwanathan, AT\&T Bell Labs Dr. S. Keshav, AT\&T Bell Labs, and Dr. Inder Gopal, IBM Watson Research Center for their useful suggestions during their visit to the ERNET Project, IISc.

\section{REFERENCES}

[DQBD Draft] Project 802 - Local and Metropolitan Area Network "Proposed Standard: Distributed Queue Dual Bus (DQDB) - Draft D12 1990"

[Newman et al.,1988] R M Newman, Z L Budrikis and J L Hullet, "The QPSX Man", IEEE Comm. Magazine, April 1988.

[Newman et al.,1986] R. M. Newman and J. L. Hullet, "Distributed Queueing: A Fast and Efficient Packet Access Protocol for QPSX", Proceedings of the Eighth International COnference on Computer Communication, Munich, pp 294-299, Elsevier Science Publishers, Sept. 1986.

[Pillai et al.] R. R. Pillai, H. Adiseshu, U. Mukherji and V. Sharma, "Distributed Queue Dual Bus Metropolitan Area Network: A Review of Per- 
formance Analysis of the Medium Access Control Protocol," Jl. of IETE Special Issue on Telematics, Vol. 39, No. 2, pp. 111-124, Mar-Apr. 1993.

[Byrne et al.] W. R. Byrne, G. W. R. Luderer, G. Clapp, B. L. Nelson, H. J. Kafka, "Evolution of Metropolitan Area Network to Broadband ISDN", IEEE Comm. Magazine, Vol. 29, No. 1, pp. 69-82, January 1991.

[IEEE Network] IEEE Network, Vol. 7, No. 2, March 1993.

[Takase at al.] A Takase, J. Yaagi, Y. Sakurai and Y. Miyamori, "An Experimental B-ISDN System for MAN Application Study", GLOBECOM 91, pp. 2100-2104, 1991.

[Altera Databook] Altera EPLD databook, 1993

[IEEE JASC] IEEE Journal on Selected Areas in Communication, 93.

[Davie] B S Davie, "The Architecture and Implementation of a High-Speed Host Interface", IEEE Journal on Selected Areas in Communication, Vol. 11, No. 2, pp 228-239, February 93.

[Traw et al.] C B Traw and J M Smith "Hardware/Software Organization of a High-Performance ATM Host Interface" IEEE Journal on Selected Areas in Communication, Vol. 11, No. 2, pp 240-253, Feb. 93.

[Solari] Edward Solari, “AT BUS Design” Annabooks, 1990.

[TAXI Datasheet] “Am7968/Am7969 TAXI chip” AMD Data Sheet, 1987.

[ODL Datasheet] "ODL 200 Lightwave Data Link with Flag" - AT\&T Data Sheet, 1988.

\section{BIOGRAPHIES}

Lakshman Narayanswamy has a B.E. from the University of Bombay and a M.S. from the University of Virginia, both in Electrical Engineering. He has been with the ERNET Project since 1989, where he is involved in Network management, networking software and hardware development.

Jasvinder Singh has a B.E. from Delhi College of Engineering, University of Delhi and M.Tech. from the University of Delhi. He worked from Feburary 1991 to December 1992 as Scientist/Engineer Grade 'SC' with the Department of Electronics. He has been with the ERNET Project, Indian Institute of Science since Dec 1992 where he is involved in the ERNET's network admistration, hardware and software development.

Umesh Kulkarni is a M.E. student in the Electrical Communication Engg department, Indian Institute of Science. He got his B.E. from Walchand College of Engg, Sangli. 
Keerthi Mitra got his B.E. from Malnad College of Engineering, Hassan. He worked at the ERNET Project from 1990 to August 1994. He is currently working with Deutsche Software Indian Ltd.

Anurag Kumar has a B.Tech. in E.E. from the Indian Institute of Technology at Kanpur, and a $\mathrm{PhD}$ from Cornell University. He was a Member of Technical Staff at AT\&T Bell Labs, Holmdel for over 6 years. Since 1988 he has been with the Indian Institute of Science (IISc), Bangalore, in the Dept. of Electrical Communication Engineering, where he is now Associate Professor. He is also the Coordinator at IISc of the Education and Research Network Project, which has set up a country-wide computer network for academic and research institutions, and conducts $R \& D$ in the area of communication networks. His own research interests are in the area of modelling, analysis, control and optimisation problems arising in communication networks and distributed systems.

Utpal Mukherji received his B.Tech. degree in electrical engineering from the Indian Institute of Technology, Bombay, in 1980 and the S.M., E.E., and Sc.D. degrees in electrical and computer science from the Massachusetts Institutes of Technology in 1982, 1984, and 1986, respectively. He was a Member of Technical Staff at AT\&T Bell Laboratories, Murray hill, from 1986 to 1989. Since 1989, he has been as Assistant professor in the Department of Electrical Communication Engineering at the Indian Institute of Science, Bangalore. His research interests are in communication networks. 